\title{
Révision 2011 des fiches Piratome et Piratox
}

\section{1 revision of the Piratome and Piratox files}

\author{
F.J. Baud \\ (C) SRLF et Springer-Verlag France 2011
}

Dans le cadre de la mise à jour du plan Vigipirate défini par les circulaires 700 et 800 du Secrétariat Général à la défense nationale (SGDN) devenu le Secrétariat général de la défense et de la sécurité nationale (SGDSN), la Direction générale de la santé a demandé à l'Agence française de sécurité sanitaire des produits de santé (Afssaps) de procéder à la révision des fiches concernant les toxiques et l'utilisation des antidotes qui pourraient être nécessaires à la prise en charge de victimes d'accidents radionucléaires ou chimiques résultant principalement d'un acte de malveillance ou d'un accident industriel. La lettre de mission définissait les membres $\mathrm{du}$ groupe de travail qui réunissait les différents horizons impliqués dans la prise en charge médicale de telles victimes. Pour un temps, médecins responsables de Samu, urgentistes préhospitaliers, hospitaliers et des services d'incendie et de secours, anesthésistes-réanimateurs chirurgicaux et réanimateurs médicaux, spécialistes en radioprotection (Institut de Radioprotection et de Sûreté Nucléaire, IRSN), toxicologues des centres antipoisons et pharmaciens, blancs, rouges et kakis ont non seulement cohabités mais se sont réunis dans une ambiance chaleureuse où les aspects médicaux et techniques ont constamment prévalu.

Le champ d'action du groupe était délimité par une lettre de mission. Inutile donc de rechercher dans ces fiches des détails sur les méthodes sophistiquées de décontamination des hommes et des matériels qui font l'objet d'un autre groupe de travail. Par contre, il a semblé essentiel au groupe de travail de rappeler dans chaque fiche que le simple déshabillage des personnes suspectes d'un accident chimique et la conservation de leurs vêtements et de leurs effets personnels dans des sacs plastiques fermés étaient deux mesures simples à la fois souvent oubliées mais efficaces pour prévenir aussi bien la poursuite de l'absorption par le sujet porteur que le transfert de contamination d'une victime d'un accident chimique. De même bien qu'intitulé

F.J. Baud ( $\square)$

Réanimation médicale et toxicologique, hôpital Lariboisière, université Paris-Diderot, F-75205 Paris cedex 13, France e-mail : Frederic.baud@lrb.aphp.fr pour moitié Piratome, le groupe n'a pas été saisi de l'étude de la prescription d'iode en cas de contamination par l'iode radioactif, ce qui est regrettable, car les problèmes des civils ne coïncident pas avec les problèmes traités par une approche militaire, problème amplement démontré par la catastrophe de Fukushima de survenue postérieure à la clôture de ces fiches.

L'ouverture d'esprit du groupe a permis de faire naître ou de développer certains concepts :

- dans un premier temps, les " armes chimiques » étaient les seules " substances d'intérêt ». En fait, ces substances sont prohibées dans leur production, leur stockage et leur utilisation. Cependant, le démantèlement de l'ex-URSS a donné naissance à un trafic de nombreuses armes, y compris des armes chimiques et des substances radioactives, contrôlé par la mafia de l'Europe de l'Est. Il existe de plus un certain simulacre dans la mesure où certaines de ces substances faisant partie des armes chimiques sont utilisées dans un but industriel, en production massive, comme le chlore et le cyanure. À titre d'exemple dans un pays, un train chargé de 500 t de cyanure part chaque semaine d'un site industriel pour être utilisé dans des synthèses organiques par d'autres industries. Nous avons pu ainsi faire prévaloir les préoccupations du civil qui pourrait être confronté à une classe de produits dénommée " produits industriels commercialisés », nom donné à des atomes ou molécules faisant l'objet d'une production quantitativement importante par l'industrie chimique et qui peuvent être achetés sans que la commande donne lieu à une surveillance ou à une autorisation particulière. Tant leur production importante que leur accessibilité en font des agents potentiels aussi bien d'incidents, voire de catastrophes industrielles que d'agents de substitution dans l'hypothèse d'une utilisation malveillante. Cette ouverture en termes de produits potentiellement concernés impacte non pas tant les traitements antidotiques qui seront mis en œuvre et qui restent qualitativement les mêmes, mais elle oblige à moduler les protocoles d'administration de ces antidotes. Ainsi, dans le cas de l'attentat au sarin de 
Tokyo, une seule dose de $2 \mathrm{mg}$ d'atropine a été suffisante pour corriger les symptômes présentés par les centaines de personnes modérément intoxiquées. Mais il est hautement improbable qu'une telle dose d'atropine suffise en cas d'intoxication par certains insecticides organophosphorés très toxiques et disponibles en raison de la durée connue pour être prolongée des symptômes qu'ils peuvent induire ;

- le principe d'utilisation en urgence des fiches a été retenu. En conséquence de quoi, tant la rédaction que le contenu de ces fiches ont été tissés en fonction de cet impératif. Inutile donc de chercher la physiopathologie, le mode d'action des toxiques et de leurs antidotes. Au maximum un mot pour résumer ce qui dans d'autres ouvrages ferait l'objet de chapitres. Le style cursif et lapidaire est voulu sans jamais prêter à confusion, nos collègues pharmaciens ne nous le permettraient pas. Par contre, il a paru important au groupe de faire apparaître certaines propriétés physicochimiques ayant un impact sur le danger : c'est ainsi que l'état physique du toxique à la température ambiante permet de séparer les agents en phase uniquement gazeuse des liquides à tension de vapeur élevée. La densité par rapport à l'air témoigne de la capacité d'un gaz à la fois à stagner en l'absence de ventilation et à créer un effet non spécifique d'anoxie de confinement en plus du risque toxique spécifique. Enfin, l'hydrosolubilité donne une indication sur l'efficacité d'une décontamination par douche simple durant cinq minutes, d'autant plus faible que le toxique sera faiblement hydrosoluble ;

- qu'il s'agisse de risque radiologique, nucléaire ou chimique, il a été tenu compte de la réalité de la situation de toute intoxication avec ses deux phases : une phase d'incertitude au cours de laquelle des décisions thérapeutiques doivent être prises, car c'est à une phase précoce, éventuellement même asymptomatique, que les antidotes et chélateurs auront leur maximum d'effet, puis une phase de certitude où les traitements peuvent être réduits, éventuellement changés et rentrés dans le cadre de protocoles bien connus. Cette phase d'incertitude aboutit tout naturellement à proposer en première intention des antidotes qui, à efficacité équivalente, induisent le moins d'effets adverses ou des effets adverses moins graves. Notons que la durée de la phase d'incertitude est elle-même... incertaine, pouvant durer moins d'une heure pour des agents facilement identifiables à des jours, des semaines (voir plus bas l'affaire Litvinenko)... voire ne jamais aboutir à une conclusion, ce qui est loin d'être exceptionnel (cf. le(s) produit(s) utilisé(s) dans l'opération de contre-terrorisme de l'opéra de Moscou);

- bien que ce point ait fait l'objet d'âpres discussions, l'absence d'antidotes et les trous de connaissance ont été précisés autant que de besoin. Le principal argument des tenants de l'abstention tenait au fait que de tels trous de connaissance pourraient inspirer des esprits malveillants. Les deux arguments des tenants de leur identification prenaient en compte le fait que la malveillance suppose une connaissance facilement acquise par les moyens d'information actuels et qu'à l'inverse, il fallait épargner à l'urgentiste de se trouver dans une situation de crise à une heure avancée de la nuit à la recherche d'un hypothétique antidote qui n'existe pas.

Dans le domaine Piratome, du radiologique et du nucléaire, le groupe a voulu une systématisation des situations vraisemblables. Cette réflexion a mis en exergue quatre scénarii crédibles aux yeux des spécialistes, à savoir :

- l'irradiation externe telle qu'elle pourrait être réalisée en plaçant une source de rayonnement sous un siège de transport en commun ;

- l'accident de centrale nucléaire ;

- l'utilisation de bombes sales ;

- l'épandage de radionucléides.

Bien que le Polonium 210 soit cité dans la liste des radionucléides d'intérêt et malgré l'histoire de l'espion russe Litvinenko, le groupe n'a pas souhaité individualiser ce cas en scénario. En effet, ce cas mixte de façon exceptionnelle les problèmes de contamination interne et ceux d'un syndrome majeur d'irradiation qui sont habituellement séparés. Ce cas mérite cependant un commentaire qui a valeur d'avertissement. Alors même que les médecins anglais étaient face à un tableau typique d'irradiation majeure, le diagnostic de certitude n'a été posé qu'au $21^{\mathrm{e}}$ jour. Pourquoi si tard ? Les médecins y avaient pensé et avaient passé le patient à un compteur, mais celui-ci ne détectait que le rayonnement gamma et non le rayonnement alpha produit par le Polonium 210 !!! La conclusion de cette malheureuse affaire est que la dosimétrie active est affaire de spécialistes du nucléaire, et tenter de se substituer à leur connaissance expose à des erreurs majeures. Cela nous a appris qu'il nous faut autant connaître les trous de connaissance qu'admettre les limites de nos connaissances.

Le premier scénario pose le problème essentiel de localisation et de neutralisation de la source en même temps que la prise en charge des victimes touchées par le syndrome d'irradiation externe. Dans la mesure où le traitement de ce syndrome ne fait jamais appel au moindre antidote, il n'a pas été développé.

Pour les trois autres scénarii, les problèmes sont :

- en plus de la contamination externe qu'il faudra mesurer et traiter par une décontamination externe ;

- le traitement chirurgical des blessés par explosion (centrale nucléaire ou bombe sale) : la primauté du geste chirurgical sur l'évaluation et le traitement de la contamination et de l'irradiation doit être rappelée ; 
- l'administration d'un agent chélateur. Ces médicaments visent :

- pour tous les radionucléides qui ont la chance de posséder un moyen thérapeutique permettant d'augmenter leur élimination, l'administration d'un traitement, généralement un chélateur, a pour but de diminuer la contamination interne. La préoccupation essentielle à moyen et long termes est de diminuer le risque de cancer radio-induit. Rappelons que d'une façon générale ces isotopes sont classés " cancérogène, mutagène et reprotoxique (CMR) » et qu'ils ont des effets dits "sans seuil », à la différence des médicaments pour lesquels il faut atteindre une dose toxique minimale pour faire apparaître un effet toxique. Toute diminution de la dose interne fait espérer un gain sur la probabilité de survenue d'un cancer ;

- pour certains radionucléides, une toxicité systémique s'ajoute aux effets des radiations ionisantes : toxicité neurologique et des phanères pour le thallium, néphrotoxicité de l'uranium, du cadmium, dysfonction multiple d'organes avec l'arsenic... Le chélateur aura pour but de diminuer, voire de protéger de cette toxicité d'organe.

Cela permet d'ailleurs de rappeler qu'un agent chélateur d'un atome l'est quelle que soit sa forme isotopique : stable ou instable. La capacité de complexation d'un chélateur est la même, que l'atome soit radioactif ou non.

Huit radionucléides font l'objet d'une attention particulière, mais il a semblé impossible au groupe de se limiter à ces seuls éléments sans offrir aux médecins qui pourraient être amenés à prendre en charge des victimes d'un accident nucléaire une information complète sur radionucléides et chélateurs. C'est ainsi que le lecteur trouvera pour chaque radionucléide connu l'existence ou non d'un agent chélateur et le degré de connaissance. Dans ce domaine, le niveau de certitude est très limité et le terme de chélateur est utilisé pour qualifier des substances dont le niveau de connaissance est extrêmement variable. Toute la littérature pertinente sur ce vaste sujet a été revue et a amené le groupe à classer ces «chélateurs » selon trois niveaux de connaissance allant du plus élémentaire au plus pertinent sur le plan thérapeutique :

- complexation in vitro ;

- études chez l'animal ;

- utilisation chez l'homme.

Ces données nous semblent à même de contribuer à la prise de décision d'une éventuelle utilisation chez l'homme. Il est en effet illusoire de penser que le service médical rendu, la diminution de la probabilité de survenue d'un cancer, puisse jamais être déterminé pour ces chélateurs.
Par contre, le groupe a été d'accord pour éliminer des « antidotes » cités dans certains textes, ouvrages officiels, voire de références, mais qui, à l'analyse de spécialistes de l'urgence, apparaissaient comme de véritables «potions magiques ou remède de rebouteux » sans qu'aucune base scientifique ne supporte leur utilisation pour un radionucléide donné.

En conclusion, par rapport aux premières fiches, des modifications importantes sur les dangers et d'énormes progrès thérapeutiques ont été accomplis : par l'éventail des substances qui pourraient être impliquées, par le caractère, hélas, crédible de ces différentes menaces, par la nécessité d'une approche clinique de ces accidents, par l'augmentation significative des capacités de diagnostic analytique, par les progrès constants avec de nouveaux antidotes, de nouveaux chélateurs pas tellement plus efficaces que les anciens mais mieux tolérés, point essentiel dans la phase d'incertitude et parfois beaucoup plus facile à administrer.

J'espère que vous prendrez autant de plaisir à lire ces fiches que nous avons eu à les écrire en gardant pleine conscience du caractère temporaire de ces connaissances autant destinées à l'obsolescence qu'à leur amélioration par les découvertes à venir.

La Société de réanimation de langue française (SRLF) a décidé, avec l'accord de l'Afssaps, de publier les fiches Piratome-Piratox 2010-2011 sur son site Internet :

- La prise en charge des intoxications aux agents chimiques (fiches Piratox avec entrée par catégorie d'agent chimique) :

- fiche Piratox $n^{\circ} 1$ «Cyanures et dérivés cyanés»;

- fiche Piratox n ${ }^{\circ} 2$ « Hydrogène arsénié ou arsine»;

- fiche Piratox $n^{0} 3$ « Lewisite»;

- fiche Piratox $n^{\circ} 4$ « Organophosphorés : neurotoxiques de guerre et pesticides $»$;

- fiche Piratox n ${ }^{\circ} 5$ « Suffocants et phosphine»;

- fiche Piratox $\mathrm{n}^{\circ} 6$ " Ypérite et autres moutardes vésicantes »;

- la prise en charge des contaminations internes à divers radionucléides (fiches Piratome avec entrée par antidote, la fiche $\mathrm{n}^{\mathrm{o}} 1$ orientant le choix de l'antidote) :

- fiche Piratome $n^{\circ} 1$ « Principes de traitement et choix des antidotes $" ;$

- fiche Piratome $\mathrm{n}^{\circ} 2$ «Bleu de Prusse »;

- fiche Piratome $\mathrm{n}^{\circ} 3$ « DMSA/BAL »;

- fiche Piratome $n^{\circ} 4$ «DTPA».

Conflit d'intérêt : F.J. BAUD est expert consultant auprès de Merck Serono pour le développement international de l'hydroxocobalamine. F.J. BAUD a obtenu le financement d'une thèse d'université bourse CIFRE pour l'optimisation du traitement des intoxications organophosphorées par la pralidoxime. Approche expérimentale. 
L'Afssaps a élaboré ces fiches de recommandations thérapeutiques en collaboration avec un groupe multidisciplinaire présidé par le Pr Frédéric Baud et composé de : Alain Cazoulat, Vincent Danel, Philippe Delpech, Frédéric Dorandeu, Claude Fuilla, Robert Garnier, Olivier Hersan, Jean-René Jourdain, Lionel Lachenaud, Eric Le Carpentier, Jean-François Marsan, Jean-Marc Sapori.

La coordination scientifique et rédactionnelle de ces fiches a été réalisée par : Vincent Gazin et Fadila
Belmahdi (Afssaps, DEMEB, service de l'évaluation et de la surveillance du risque et de l'information sur le médicament - Anne Castot, département de toxicologie - Dominique Masset, unité de toxicologie clinique).

Ont également participé à la réflexion : Marianne Deschenes (Afssaps, service de coordination de l'information, des vigilances, des risques et des actions de santé publique), Catherine Rey-Quynio et Christelle Jadeau (Afssaps, DEMEB, unité PTC2). 